ORIGINAL ARTICLE

\title{
An updated study of mortality among North American synthetic rubber industry workers
}

\author{
N Sathiakumar, J Graff, M Macaluso, G Maldonado, R Matthews, E Delzell
}

Occup Environ Med 2005;62:822-829. doi: 10.1136/oem.2004.018176

See end of article for authors' affiliations

Correspondence to: Dr N Sathiakumar, School of Public Health, University of Alabama at

Birmingham, 1665

University Boulevard,

Birmingham, AL 35294,

USA; nalini@uab.edu

Accepted 10 July 2005
Aim: This study evaluated the mortality experience of workers from the styrene-butadiene industry.

Methods: The authors added seven years of follow up to a previous investigation of mortality among 17924 men employed in the North American synthetic rubber industry. Analyses used the standardised mortality ratios (SMRs) to compare styrene-butadiene rubber workers' cause specific mortality (1943-98) with those of the United States and the Ontario general populations.

Results: Overall, the observed/expected numbers of deaths were $6237 / 7242$ for all causes (SMR $=86$, $95 \% \mathrm{Cl} 84$ to 88 ) and 1608/1741 for all cancers combined (SMR=92,95\% Cl 88 to 97), 71/61 for leukaemia, 53/53 for non-Hodgkin's lymphoma, and 26/27 for multiple myeloma. The $16 \%$ leukaemia increase was concentrated in hourly paid subjects with 20-29 years since hire and 10 or more years of employment in the industry $(19 / 7.4, \mathrm{SMR}=258,95 \% \mathrm{Cl} 156$ to 403$)$ and in subjects employed in polymerisation (18/8.8, SMR $=204,95 \% \mathrm{Cl} 121$ to 322 ), maintenance labour (15/7.4, SMR =326, 95\% $\mathrm{Cl} 178$ to 456$)$, and laboratory operations (14/4.3, SMR $=326,95 \% \mathrm{Cl} 178-546)$.

Conclusion: The study found that some subgroups of synthetic rubber workers had an excess of mortality from leukaemia that was not limited to a particular form of leukaemia. Uncertainty remains about the specific agent(s) that might be responsible for the observed excesses and about the role of unidentified confounding factors. The study did not find any clear relation between employment in the industry and other forms of lymphohaematopoietic cancer. Some subgroups of subjects had more than expected deaths from colorectal and prostate cancers. These increases did not appear to be related to occupational exposure in the industry.
W e previously evaluated the mortality experience of 17964 North American synthetic rubber industry workers during the period 1944 through $1991 . .^{1-4}$ The workers had an increased rate of death from leukaemia that was concentrated among hourly paid men with 20 or more years since hire and 10 or more years of employment in the industry and in subjects employed in polymerisation, maintenance labour, and laboratories, three areas in styrenebutadiene operations with potential for relatively high exposure to the monomers 1,3-butadiene (butadiene) and styrene and the polymerisation shortstopping agent dimethyldithiocarbamate (DMDTC). Although benzene was used in some synthetic rubber facilities, this agent did not appear be associated with mortality from leukaemia in the industry. ${ }^{2}$ The research described here adds seven years of follow up to this study and evaluates the 1944-98 mortality experience of the study group in relation to time since hire, work duration, and work area. A companion paper will describe the effect of butadiene, styrene, and DMDTC exposures on lymphohaematopoietic cancer (LHC) and other diseases.

\section{METHODS}

In previous publications, we described plant operations and the methods used to identify subjects, to develop work histories, and to determine vital status for the original follow up period of 1944-91. ${ }^{1-3}$ The original study included 17964 men classified as having worked, before 1 January 1992, for at least one year at any of eight synthetic rubber plants, seven in the US and one in Canada. The updated investigation included 17924 of these subjects. The number of subjects decreased because we combined the work histories of 31 men in the original study who had worked at two different plants and had two separate sets of records, we excluded eight men (five from US plants, three from the Canadian plant) who had worked for slightly less than one year, and we found that one subject was a woman.

Information available from the original study included name, social security number (for US subjects), birth date, race, vital status as of the end of 1991, death date for those dying in or before 1991, and work history through the end of 1991. We did not obtain any additional, recent work history information for the 3763 subjects who were still active as of the end of 1991.

We used work histories to classify subjects according to employment in five major work areas: production, maintenance, labour, laboratories, and other operations. ${ }^{1}$ For six of the eight plants, work histories were sufficiently detailed to permit specification of three work area subgroups of production (polymerisation, coagulation, finishing), two subgroups of maintenance (shop maintenance, field maintenance), and two subgroups of labour (production labour, maintenance labour). Work histories provided information on each job held by a subject, including the start and end dates, a text description of the work area and job, and the payroll classification (hourly or salaried) of the position.

The original study identified 4665 subjects as deceased before 1992, 12565 as presumed living, and 734 (US plants) as having unknown vital status. ${ }^{1}$ We updated vital status information through 1998 for the study group. Vital status information was obtained for US subjects by linkages with Social Security Administration (SSA) data, Pension Benefits Information records, and the National Death Index (NDI)

Abbreviations: CMDB, Canadian Mortality Data Base; DMDTC, dimethyldithiocarbamate; ICD, International Classification of Diseases; LHC, lymphohaematopoietic cancer; NDI, National Death Index; NHL, non-Hodgkin's lymphoma; SMR, standardised mortality ratio 
Table 1 Number of subjects by selected characteristics for white and non-white men and all subjects combined

\begin{tabular}{|c|c|c|c|c|c|c|}
\hline & \multicolumn{2}{|l|}{ White } & \multicolumn{2}{|c|}{ Non-white } & \multicolumn{2}{|l|}{ Total } \\
\hline & $n$ & (\%) & $\mathrm{n}$ & (\%) & $n$ & (\%) \\
\hline Total & 15583 & $(100)$ & 2341 & $(100)$ & 17924 & $(100)$ \\
\hline \multicolumn{7}{|c|}{ Plant, location, follow up start year } \\
\hline 1, Kentucky, 1965 & 1237 & (8) & 154 & (7) & 1391 & (8) \\
\hline 2, Texas, 1944 & 664 & (4) & 112 & (5) & 776 & (4) \\
\hline 3, Louisiana, 1944 & 1510 & (10) & 479 & (20) & 1989 & (11) \\
\hline 4, Lovisiana, 1944 & 1542 & (10) & 542 & (23) & 2084 & (12) \\
\hline 5, Texas, 1960 & 427 & (3) & 136 & (6) & 563 & (3) \\
\hline 6, Texas, 1944 & 1955 & (13) & 373 & (16) & 2328 & (13) \\
\hline 7, Ontario, 1950 & 5356 & (34) & - & - & 5356 & (30) \\
\hline 8, Texas, 1944 & 2892 & (19) & 545 & (23) & 3437 & (19) \\
\hline \multicolumn{7}{|l|}{ Payroll status } \\
\hline Ever hourly & 12732 & (82) & 2278 & (97) & 15010 & (84) \\
\hline Never hourly & 2851 & (18) & 63 & (3) & 2914 & (16) \\
\hline \multicolumn{7}{|l|}{ Year of hire } \\
\hline$<1950$ & 4109 & (26) & 547 & (23) & 4656 & (26) \\
\hline $1950-59$ & 4333 & (28) & 567 & (24) & 4900 & (27) \\
\hline 1960-69 & 3338 & (21) & 419 & (18) & 3757 & (21) \\
\hline $1970+$ & 3803 & (24) & 808 & (35) & 4611 & (26) \\
\hline Median & 1958 & & 1962 & & 1958 & \\
\hline \multicolumn{7}{|c|}{ Years worked at end of 1991} \\
\hline$<10$ & 7223 & (46) & 1086 & (46) & 8309 & (46) \\
\hline $10-19$ & 3325 & (21) & 540 & (23) & 3865 & (22) \\
\hline $20+$ & 5035 & (32) & 715 & (31) & 5750 & (32) \\
\hline Median & 11 & & 12 & & 11 & \\
\hline \multicolumn{7}{|c|}{ Years since hire at end of follow up } \\
\hline$<20$ & 2990 & (19) & 521 & (22) & 3511 & (20) \\
\hline $20-29$ & 3494 & (22) & 765 & (33) & 4259 & (24) \\
\hline $30+$ & 9099 & (58) & 1055 & (45) & 10154 & (57) \\
\hline Median & 33 & & 29 & & 33 & \\
\hline \multicolumn{7}{|l|}{ Vital status } \\
\hline Alive & 9787 & (63) & 1330 & (57) & 11117 & (62) \\
\hline Deceased & 5384 & (35) & 853 & (36) & 6237 & (35) \\
\hline Unknown & 412 & (3) & 158 & (7) & 570 & (3) \\
\hline \multicolumn{7}{|c|}{ Age at end of study (years) } \\
\hline$<60$ & 6696 & (43) & 1251 & (53) & 7947 & (44) \\
\hline $60-69$ & 4272 & (27) & 536 & (23) & 4808 & (27) \\
\hline $70-79$ & 3217 & (21) & 414 & (18) & 3631 & (21) \\
\hline $80+$ & 1398 & (9) & 140 & (6) & 1538 & (9) \\
\hline Median & 62 & & 58 & & 62 & \\
\hline
\end{tabular}

and by individual tracing and, for Canadian subjects, by linkage with the Canadian Mortality Data Base (CMDB) of Statistics Canada. Using the above procedures, we established the vital status of $97 \%$ of the study group. Only 570 subjects $(3 \%)$ were lost to follow up.

We used several procedures to determine the International Classification of Diseases (ICD) code for decedents' underlying cause of death. For US subjects who died in or after 1979, we used codes from the NDI-Plus. For US deaths before 1979 a nosologist assigned an ICD code to the underlying cause of death. Cause of death codes for Canadian decedents came from the CMDB. All codes for US and Canadian decedents were based on the revision of the ICD in effect at the time of death.

We compared subjects' mortality rates to the rates of the male general population of the states where the US plants were located (Texas, Kentucky, or Louisiana) (US subjects) or to the rates of the male general population of Ontario (Canadian subjects). For most analyses, the beginning of follow up varied by plant from 1944-65, depending on the availability of employment records, ${ }^{3}$ and ended on 31 December 1998.

We used the standardised mortality ratio (SMR) as the measure of association for comparing the mortality rates of all subjects combined and of subgroups specified by the basis of work area and other employment factors, with the rates of the state and provincial general populations. Employment factors included duration of employment computed until the date of last known employment and time since hire computed based on the start date of employment in the industry. We computed the SMR for a particular cause of death as the ratio $(\times 100)$ of the number of deaths observed among subjects to the number expected based on the cause, race, age, and calendar time specific mortality rates of state or Ontario general populations. To compute expected numbers of deaths, we first accumulated person-years of observation for each subject into race specific five year age and calendar time categories. Person-year accumulation began on the date when the subject accumulated one year of employment, on the date of entry into a particular subject subgroup, or on the date when personnel records at the subject's plant were considered complete, whichever was latest. Person-year accumulation ended on the date of death, on the date of loss to follow up (the termination date for lost subjects), or on the study closing date (31 December 1998), whichever was earliest. Next, we multiplied the race, age, and calendar time specific person-years by the corresponding state or Ontario mortality rates and summed the resulting quantities to obtain expected numbers. We used the Occupational Mortality Analysis Program (OCMAP) ${ }^{5}$ to compute SMRs and their $95 \%$ confidence intervals.

We also carried out analyses of specific forms of leukaemia. For these analyses, we limited the follow up period to 1968 through 1998 for US subjects and to 1969 through 1998 for Canadian subjects. Before these time periods, the ICD coding system used for general population rates did not distinguish 
Table 2 Observed/expected number of deaths, SMR, and $95 \% \mathrm{Cl}$ by calendar period of follow up, all subjects

\begin{tabular}{|c|c|c|c|c|c|c|c|c|c|}
\hline \multirow[b]{2}{*}{ Cause of death } & \multicolumn{3}{|l|}{$1944-91$} & \multicolumn{3}{|l|}{ 1992-98 } & \multicolumn{3}{|l|}{$1944-98$} \\
\hline & Obs/exp & SMR & $(95 \% \mathrm{Cl})$ & Obs/exp & SMR & $(95 \% \mathrm{Cl})$ & Obs/exp & SMR & $(95 \% \mathrm{Cl})$ \\
\hline All causes & $4659 / 5490.4$ & 85 & $(82-87)$ & $1578 / 1751.9$ & 90 & $(86-95)$ & $6237 / 7241.8$ & 86 & $(84-88)$ \\
\hline All cancer & $1116 / 1237.9$ & 90 & $(85-96)$ & $492 / 503.4$ & 98 & (89-107) & $1608 / 1741.2$ & 92 & (88-97) \\
\hline Buccal cavity \& pharynx & $18 / 36.0$ & 50 & (30-79) & $4 / 11.0$ & 36 & (10-93) & $22 / 47.0$ & 47 & (29-71) \\
\hline Oesophagus & $25 / 32.5$ & 77 & $(50-114)$ & $19 / 14.3$ & 133 & (80-208) & $44 / 46.8$ & 94 & $(68-126)$ \\
\hline Stomach & $48 / 60.5$ & 79 & $(59-105)$ & $16 / 14.9$ & 107 & $(61-174)$ & $64 / 75.4$ & 85 & $(65-108)$ \\
\hline Colorectum & $138 / 125.7$ & 110 & $(92-130)$ & $55 / 52.1$ & 106 & (80-137) & $193 / 177.8$ & 109 & (94-125) \\
\hline Liver & $17 / 27.6$ & 62 & $(36-99)$ & $15 / 15.0$ & 100 & $(56-165)$ & $32 / 42.6$ & 75 & $(51-106)$ \\
\hline Pancreas & $49 / 64.4$ & 76 & $(56-101)$ & $27 / 23.4$ & 116 & $(76-168)$ & $76 / 87.8$ & 87 & (68-108) \\
\hline Larynx & $17 / 17.7$ & 96 & $(56-54)$ & $0 / 6.4$ & 0 & $(0-57)$ & $17 / 24.1$ & 71 & $(41-113)$ \\
\hline Lung & $406 / 438.3$ & 93 & $(84-102)$ & $157 / 180.2$ & 87 & $(74-102)$ & $563 / 618.4$ & 91 & (84-99) \\
\hline Prostate & $91 / 92.8$ & 98 & $(79-120)$ & $63 / 55.8$ & 113 & (87-145) & $154 / 148.6$ & 104 & $(88-121)$ \\
\hline Bladder & $26 / 29.2$ & 89 & $(58-131)$ & $11 / 11.8$ & 93 & $(47-167)$ & $37 / 41.0$ & 90 & $(64-125)$ \\
\hline Kidney & $26 / 28.4$ & 92 & $(60-134)$ & $13 / 12.2$ & 107 & (57-182) & $39 / 40.6$ & 96 & $(68-131)$ \\
\hline Brain & $28 / 30.1$ & 93 & $(62-135)$ & $9 / 10.0$ & 90 & $(41-172)$ & $37 / 40.0$ & 93 & $(65-128)$ \\
\hline $\mathrm{LHC}$ & $115 / 108.0$ & 107 & $(88-128)$ & $47 / 45.1$ & 104 & $(77-139)$ & $162 / 153.1$ & 106 & $(90-123)$ \\
\hline $\mathrm{NHL}$ & $33 / 35.4$ & 93 & (64-131) & $20 / 17.9$ & 112 & $(68-173)$ & $53 / 53.3$ & 100 & $(75-130)$ \\
\hline Hodgkin's disease & $11 / 9.8$ & 113 & $(56-202)$ & $1 / 1.0$ & 98 & $(2-545)$ & $12 / 10.8$ & 111 & (58-195) \\
\hline Leukaemia & $51 / 44.0$ & 116 & (86-153) & $20 / 17.9$ & 117 & (71-181) & $71 / 61.1$ & 116 & (91-147) \\
\hline Multiple myeloma & $20 / 18.1$ & 110 & $(67-170)$ & $6 / 9.1$ & 66 & $(24-143)$ & $26 / 27.3$ & 95 & $(62-140)$ \\
\hline Other cancer & $112 / 147.4$ & 76 & $(63-92)$ & $56 / 51.4$ & 109 & $(82-141)$ & $168 / 197.6$ & 85 & $(73-99)$ \\
\hline Benign neoplasms & $11 / 12.2$ & 90 & $(45-162)$ & $5 / 3.9$ & 130 & $(42-302)$ & $16 / 16.0$ & 100 & $(57-162)$ \\
\hline Blood disorders & $18 / 19.0$ & 95 & $(56-150)$ & $5 / 10.5$ & 48 & $(16-111)$ & $23 / 29.4$ & 78 & $(50-117)$ \\
\hline Mental disorders & $15 / 38.8$ & 39 & $(22-64)$ & $21 / 22.8$ & 92 & $(57-141)$ & $36 / 61.6$ & 58 & $(41-81)$ \\
\hline Allergic, endocrine, \& metabolic disease & $74 / 107.8$ & 69 & $(54-86)$ & $48 / 64.4$ & 75 & $(55-99)$ & $122 / 172.2$ & 71 & $(59-85)$ \\
\hline Nervous system disease & $57 / 60.7$ & 94 & $(71-122)$ & $38 / 35.5$ & 107 & $(76-147)$ & $95 / 96.1$ & 99 & $(80-121)$ \\
\hline Circulatory disease & $2303 / 2569.1$ & 90 & $(86-93)$ & $629 / 712.8$ & 88 & $(82-95)$ & $2932 / 3281.8$ & 89 & $(86-93)$ \\
\hline Non-malignant respiratory disease & $248 / 351.5$ & 71 & $(62-80)$ & $146 / 160.6$ & 91 & (77-107) & $394 / 512.1$ & 77 & $(70-85)$ \\
\hline Digestive disease & $146 / 233.3$ & 63 & $(53-74)$ & $41 / 62.3$ & 66 & $(47-89)$ & $187 / 295.5$ & 63 & $(55-73)$ \\
\hline Genitourinary disease & $55 / 87.3$ & 63 & $(48-82)$ & $21 / 30.3$ & 69 & $(43-106)$ & $76 / 117.6$ & 65 & $(51-81)$ \\
\hline External causes & $370 / 572.8$ & 65 & $(58-72)$ & $76 / 82.2$ & 92 & $(73-116)$ & $446 / 655.1$ & 68 & $(62-75)$ \\
\hline Other known & $129 / 198.5$ & 65 & $(54-77)$ & $49 / 64.5$ & 76 & (57-101) & $178 / 262.8$ & 68 & $(58-78)$ \\
\hline Unknown & 117 & & & 7 & & & 124 & & \\
\hline
\end{tabular}

among acute lymphocytic, acute myelogenous, and other acute forms of leukaemia but, instead, combined all acute leukaemias into a single category. The earlier coding systems also did not distinguish between chronic myelogenous leukaemia and myelogenous leukaemia unspecified as acute or chronic or between chronic lymphocytic leukaemia and

Table 3 Observed/expected number of deaths, SMR, and $95 \% \mathrm{Cl}$ for selected cancers and selected subgroups, 1944-98

\begin{tabular}{|c|c|c|c|c|c|c|c|}
\hline & & $\begin{array}{l}\text { Colorectal } \\
\text { cancer }\end{array}$ & $\begin{array}{l}\text { Prostate } \\
\text { cancer }\end{array}$ & $\begin{array}{l}\text { Hodgkin's } \\
\text { disease }\end{array}$ & $\begin{array}{l}\text { Multiple } \\
\text { myeloma }\end{array}$ & All leukaemia & NHL \\
\hline \multirow[t]{2}{*}{ All subjects } & Obs/exp & $193 / 177.8$ & $154 / 148.6$ & $12 / 10.8$ & $26 / 27.3$ & $71 / 61.1$ & $53 / 53.3$ \\
\hline & $\begin{array}{l}\text { SMR } \\
(95 \% \mathrm{Cl})\end{array}$ & $\begin{array}{l}109 \\
(94-125)\end{array}$ & $\begin{array}{l}104 \\
(88-121)\end{array}$ & $\begin{array}{l}111 \\
(58-195)\end{array}$ & $\begin{array}{l}95 \\
(62-140)\end{array}$ & $\begin{array}{l}116 \\
(91-147)\end{array}$ & $\begin{array}{l}100 \\
(75-130)\end{array}$ \\
\hline \multirow[t]{3}{*}{ Ever hourly } & Obs/exp & $161 / 150.0$ & $130 / 127.8$ & $7 / 9.1$ & $20 / 23.2$ & $63 / 51.3$ & $49 / 44.2$ \\
\hline & SMR & 107 & 102 & 77 & 86 & 123 & 111 \\
\hline & (95\% Cl) & $(91-125)$ & $(85-121)$ & $(31-158)$ & (53-133) & (94-157) & $(82-147)$ \\
\hline \multirow{3}{*}{ Never hourly } & Obs/exp & $32 / 27.8$ & $24 / 20.8$ & $5 / 1.6$ & $6 / 4.1$ & $8 / 9.8$ & $4 / 9.1$ \\
\hline & SMR & 115 & 115 & 305 & 146 & 82 & \\
\hline & $(95 \% \mathrm{Cl})$ & (79-162) & $(74-172)$ & (99-711) & $(54-317)$ & $(35-161)$ & $(12-112)$ \\
\hline \multicolumn{8}{|c|}{$\begin{array}{l}\text { All hourly subjects by years since hire } \\
\text { (ysh) and years worked (yrs) }\end{array}$} \\
\hline$<20$ ysh, & Obs/exp & $10 / 12.5$ & $1 / 5.2$ & $2 / 3.3$ & $0 / 1.6$ & $4 / 7.0$ & $0 / 4.9$ \\
\hline$<10$ yrs & $\begin{array}{l}\text { SMR } \\
(95 \% \mathrm{Cl})\end{array}$ & $\begin{array}{l}80 \\
(38-147)\end{array}$ & $\begin{array}{l}19 \\
(1-108)\end{array}$ & $\begin{array}{l}61 \\
(7-221)\end{array}$ & $\begin{array}{l}0 \\
(0-236)\end{array}$ & $\begin{array}{l}57 \\
(16-146)\end{array}$ & $\begin{array}{l}0 \\
(0-76)\end{array}$ \\
\hline$<20$ ysh, & Obs/exp & $9 / 10.9$ & $3 / 4.0$ & $3 / 1.7$ & $3 / 1.5$ & $6 / 4.4$ & $1 / 3.6$ \\
\hline $10+$ yrs & $\begin{array}{l}\text { SMR } \\
(95 \% \mathrm{CI})\end{array}$ & $\begin{array}{l}83 \\
(38-157)\end{array}$ & $\begin{array}{l}75 \\
(15-218)\end{array}$ & $\begin{array}{l}178 \\
(37-519)\end{array}$ & $\begin{array}{l}207 \\
(43-605)\end{array}$ & $\begin{array}{l}136 \\
(50-296)\end{array}$ & $\begin{array}{l}28 \\
(1-155)\end{array}$ \\
\hline \multirow{2}{*}{$\begin{array}{l}20-29 \text { ysh, } \\
<10 \text { yrs }\end{array}$} & Obs/exp & $11 / 11.1$ & $7 / 7.7$ & $1 / 0.8$ & $2 / 1.7$ & $4 / 4.1$ & $5 / 3.5$ \\
\hline & $\begin{array}{l}\text { SMR } \\
(95 \% \mathrm{CI})\end{array}$ & $\begin{array}{l}99 \\
(49-177)\end{array}$ & $\begin{array}{l}90 \\
(36-186)\end{array}$ & $\begin{array}{l}129 \\
(3-720)\end{array}$ & $\begin{array}{l}117 \\
(14-423)\end{array}$ & $\begin{array}{l}98 \\
(27-251)\end{array}$ & $\begin{array}{l}143 \\
(46-333)\end{array}$ \\
\hline \multirow{2}{*}{$\begin{array}{l}20-29 \text { ysh, } \\
10+\text { yrs }\end{array}$} & Obs/exp & $35 / 23.8$ & $12 / 13.6$ & $1 / 1.4$ & $6 / 3.4$ & $19 / 7.4$ & $11 / 6.5$ \\
\hline & $\begin{array}{l}\text { SMR } \\
(95 \% \mathrm{CI})\end{array}$ & $\begin{array}{l}147 \\
(103-205)\end{array}$ & $\begin{array}{l}88 \\
(46-154)\end{array}$ & $\begin{array}{l}70 \\
(2-392)\end{array}$ & $\begin{array}{l}175 \\
(64-380)\end{array}$ & $\begin{array}{l}258 \\
(156-403)\end{array}$ & $\begin{array}{l}170 \\
(85-305)\end{array}$ \\
\hline \multirow{2}{*}{$\begin{array}{l}30+\text { ysh, } \\
<10 \text { yrs }\end{array}$} & Obs/exp & $30 / 26.5$ & $46 / 29.8$ & $0 / 0.6$ & $3 / 4.6$ & $10 / 8.9$ & $7 / 8.0$ \\
\hline & $\begin{array}{l}\text { SMR } \\
(95 \% \text { CI) }\end{array}$ & $\begin{array}{l}113 \\
(76-162)\end{array}$ & $\begin{array}{l}155 \\
(113-206)\end{array}$ & $\begin{array}{l}0 \\
(0-591)\end{array}$ & $\begin{array}{l}65 \\
(13-189)\end{array}$ & $\begin{array}{l}113 \\
(54-207)\end{array}$ & $\begin{array}{l}87 \\
(35-179)\end{array}$ \\
\hline \multirow{3}{*}{$\begin{array}{l}30+y s h \\
10+y r s\end{array}$} & Obs/exp & $66 / 65.2$ & $61 / 67.4$ & $0 / 1.4$ & $6 / 10.3$ & $20 / 19.6$ & $25 / 17.7$ \\
\hline & SMR & 101 & 90 & 0 & 58 & 102 & 141 \\
\hline & $(95 \% \mathrm{Cl})$ & (78-129) & $(69-116)$ & $(0-271)$ & $(21-126)$ & $(62-158)$ & (91-208) \\
\hline
\end{tabular}

NHL, non-Hodgkin's lymphoma; ysh, years since hire. 
Table 4 Observed/expected numbers of deaths, SMR, and 95\% CI for cell type specific leukaemia among selected subgroups, 1968/1969-98

\begin{tabular}{|c|c|c|c|c|c|c|c|c|}
\hline & & \multicolumn{3}{|c|}{ Lymphocytic leukaemia } & \multicolumn{3}{|c|}{ Myelogrenous leukaemia } & \multirow[b]{2}{*}{ Other leukaemia* } \\
\hline & & Total & Acute & Chronic & Total & Acute & Chronic & \\
\hline All subjects & $\begin{array}{l}\text { Obs/exp } \\
\text { SMR } \\
(95 \% \mathrm{Cl})\end{array}$ & $\begin{array}{l}19 / 14.8 \\
128 \\
(77-200)\end{array}$ & $\begin{array}{l}1 / 2.4 \\
42 \\
(1-234)\end{array}$ & $\begin{array}{l}16 / 10.6 \\
151 \\
(87-247)\end{array}$ & $\begin{array}{l}28 / 22.0 \\
127 \\
(84-183)\end{array}$ & $\begin{array}{l}14 / 13.7 \\
102 \\
(56-171)\end{array}$ & $\begin{array}{l}11 / 6.6 \\
167 \\
(83-299)\end{array}$ & $\begin{array}{l}18 / 14.6 \\
123 \\
(73-194)\end{array}$ \\
\hline Ever hourly & $\begin{array}{l}\text { Obs/exp } \\
\text { SMR } \\
(95 \% \mathrm{Cl})\end{array}$ & $\begin{array}{l}17 / 12.3 \\
138 \\
(80-221)\end{array}$ & $\begin{array}{l}1 / 2.0 \\
51 \\
(1-282)\end{array}$ & $\begin{array}{l}15 / 8.8 \\
171 \\
(96-281)\end{array}$ & $\begin{array}{l}24 / 18.3 \\
131 \\
(84-195)\end{array}$ & $\begin{array}{l}11 / 11.3 \\
97 \\
(48-173)\end{array}$ & $\begin{array}{l}11 / 5.5 \\
200 \\
(100-358)\end{array}$ & $\begin{array}{l}17 / 12.2 \\
139 \\
(81-222)\end{array}$ \\
\hline Never hourly & $\begin{array}{l}\text { Obs/exp } \\
\text { SMR } \\
(95 \% \mathrm{Cl})\end{array}$ & $\begin{array}{l}2 / 2.5 \\
81 \\
(10-293)\end{array}$ & $\begin{array}{l}0 / 0.4 \\
0 \\
(0-913)\end{array}$ & $\begin{array}{l}1 / 1.8 \\
57 \\
(1-319)\end{array}$ & $\begin{array}{l}4 / 3.8 \\
106 \\
(29-271)\end{array}$ & $\begin{array}{l}3 / 2.4 \\
127 \\
(26-370)\end{array}$ & $\begin{array}{l}0 / 1.1 \\
0 \\
(0-338)\end{array}$ & $\begin{array}{l}1 / 2.4 \\
42 \\
(1-231)\end{array}$ \\
\hline
\end{tabular}

lymphocytic leukaemia unspecified as acute or chronic. Person-year accumulation for this set of analyses began on the date when the subject accumulated one year of employment, on the date of entry into a particular subject subgroup, or on 1 January 1968 (US subjects) or 1 January 1969 (Canadian subjects), whichever was latest. Person-year accumulation ended as described above for the general mortality analyses. Analyses of specific forms of leukaemia included 16859 (94\%) of the 17924 total study subjects.

We present results for chronic lymphocytic leukaemia and NHL separately. Although like chronic lymphocytic leukaemia, most (about 85\%) NHLs are B-cell malignancies, for the most part they are not the same disease. ${ }^{6}$ The only exception is that chronic lymphocytic leukaemia and a rare form of NHL (small lymphocytic lymphoma, SLL) are now considered to be the same disease and together account for a very small proportion (about 5\%) of all NHLs.

Analyses of major work areas were limited to the 15612 subjects employed in SBR related operations at the eight plants. Analyses of work area subgroups were limited to the 14273 subjects employed in SBR related operations at the six plants that had detailed work histories.

\section{RESULTS}

Of the 17924 subjects, 15583 (87\%) were white and 2341 (13\%) were non-white (table 1). Most (15 010, 84\%) had worked as hourly paid employees, whereas 2914 (16\%) had never been hourly. The median plant hire year was 1958, and subjects had worked at the plants for a median of 11 years as of the end of 1991. As of the end of 1998, the median time since hire was 33 years, and the median age was 62 years. Of the 15612 men who had worked in SBR operations, 13477 $(86 \%)$ were ever hourly, and 2135 (14\%) were never hourly. Subjects accrued a total of 540586 person-years of follow up.

Of the 6237 deaths among subjects during 1944-98, 4659 (75\%) occurred in the original study period of 1944-91, and 1578 (25\%) occurred in 1992-98, the time period covered by the update (table 2 ). For the overall study group compared to state or provincial general populations, the SMR for all causes of death combined was 86 (6237 observed/7242 expected deaths, CI 84 to 88 ) and was slightly lower in 1944-91 $(\mathrm{SMR}=85, \mathrm{CI} 82$ to 87$)$ than in $1992-98(\mathrm{SMR}=90, \mathrm{CI} 86$ to 95). A similar pattern was present for all cancer deaths combined, for which the SMR was 92 (CI 88 to 97) in 194498 and was lower in 1944-91 ( SMR $=90$, CI 85 to 96) than in

\begin{tabular}{|c|c|c|c|c|c|c|c|}
\hline & & $\begin{array}{l}\text { Colorectal } \\
\text { cancer }\end{array}$ & $\begin{array}{l}\text { Prostate } \\
\text { cancer }\end{array}$ & $\begin{array}{l}\text { Hodgkin's } \\
\text { disease }\end{array}$ & $\begin{array}{l}\text { Multiple } \\
\text { myeloma }\end{array}$ & $\begin{array}{l}\text { All } \\
\text { leukaemia }\end{array}$ & NHL \\
\hline \multirow[t]{2}{*}{ Production, polymerisation } & Obs/exp & $22 / 24.6$ & $23 / 19.7$ & $0 / 1.4$ & $1 / 3.8$ & $18 / 8.8$ & $11 / 8.0$ \\
\hline & $\begin{array}{l}\text { SMR } \\
(95 \% \mathrm{Cl})\end{array}$ & $\begin{array}{l}89 \\
(56-135)\end{array}$ & $\begin{array}{l}117 \\
(74-175)\end{array}$ & $\begin{array}{l}0 \\
(0-260)\end{array}$ & $\begin{array}{l}26 \\
(1-146)\end{array}$ & $\begin{array}{l}204 \\
(121-322)\end{array}$ & $\begin{array}{l}137 \\
(69-246)\end{array}$ \\
\hline \multirow[t]{2}{*}{ Production, coagulation } & Obs/exp & $14 / 11.8$ & $9 / 9.5$ & $0 / 0.7$ & $0 / 2.0$ & $10 / 4.3$ & $4 / 4.0$ \\
\hline & $\begin{array}{l}\text { SMR } \\
\text { (95\% CI) }\end{array}$ & $\begin{array}{l}119 \\
(65-200)\end{array}$ & $\begin{array}{l}95 \\
(43-180)\end{array}$ & $\begin{array}{l}0 \\
(0-569)\end{array}$ & $\begin{array}{l}0 \\
(0-183)\end{array}$ & $\begin{array}{l}231 \\
(111-425)\end{array}$ & $\begin{array}{l}100 \\
(27-256)\end{array}$ \\
\hline Production, finishing & $\begin{array}{l}\text { Obs/exp } \\
\text { SMR } \\
(95 \% \text { CI) }\end{array}$ & $\begin{array}{l}36 / 32.4 \\
111 \\
(78-154)\end{array}$ & $\begin{array}{l}21 / 23.4 \\
90 \\
(55-137)\end{array}$ & $\begin{array}{l}2 / 2.3 \\
88 \\
(11-319)\end{array}$ & $\begin{array}{l}2 / 5.3 \\
38 \\
(5-137)\end{array}$ & $\begin{array}{l}19 / 12.2 \\
156 \\
(94-244)\end{array}$ & $\begin{array}{l}16 / 11.2 \\
143 \\
(82-233)\end{array}$ \\
\hline \multirow[t]{2}{*}{ Maintenance, shop } & Obs/exp & $16 / 11.3$ & $9 / 9.4$ & $0 / 0.7$ & $1 / 1.7$ & $4 / 4.3$ & $4 / 3.8$ \\
\hline & $\begin{array}{l}\text { SMR } \\
\text { (95\% CI) }\end{array}$ & $\begin{array}{l}142 \\
(81-231)\end{array}$ & $\begin{array}{l}95 \\
(44-181)\end{array}$ & $\begin{array}{l}0 \\
(0-530)\end{array}$ & $\begin{array}{l}57 \\
(1-320)\end{array}$ & $\begin{array}{l}93 \\
(25-238)\end{array}$ & $\begin{array}{l}105 \\
(29-268)\end{array}$ \\
\hline Maintenance, field & $\begin{array}{l}\text { Obs/exp } \\
\text { SMR } \\
(95 \% \text { CI) }\end{array}$ & $\begin{array}{l}41 / 33.5 \\
122 \\
(88-166)\end{array}$ & $\begin{array}{l}21 / 27.3 \\
77 \\
(48-118)\end{array}$ & $\begin{array}{l}1 / 1.9 \\
53 \\
(1-296)\end{array}$ & $\begin{array}{l}3 / 5.0 \\
60 \\
(12-175)\end{array}$ & $\begin{array}{l}10 / 11.9 \\
84 \\
(40-155)\end{array}$ & $\begin{array}{l}11 / 10.6 \\
104 \\
(52-186)\end{array}$ \\
\hline Labour, production & $\begin{array}{l}\text { Obs/exp } \\
\text { SMR } \\
(95 \% \text { CI) }\end{array}$ & $\begin{array}{l}12 / 10.6 \\
113 \\
(58-198)\end{array}$ & $\begin{array}{l}11 / 11.7 \\
94 \\
(47-168)\end{array}$ & $\begin{array}{l}0 / 0.7 \\
0 \\
(0-547)\end{array}$ & $\begin{array}{l}4 / 2.1 \\
189 \\
(52-483)\end{array}$ & $\begin{array}{l}4 / 3.3 \\
123 \\
(34-315)\end{array}$ & $\begin{array}{l}4 / 2.5 \\
157 \\
(43-403)\end{array}$ \\
\hline Labour, maintenance & $\begin{array}{l}\text { Obs/exp } \\
\text { SMR } \\
(95 \% \text { CI) }\end{array}$ & $\begin{array}{l}21 / 22.7 \\
93 \\
(57-141)\end{array}$ & $\begin{array}{l}31 / 24.3 \\
128 \\
(87-181)\end{array}$ & $\begin{array}{l}1 / 1.4 \\
74 \\
(2-411)\end{array}$ & $\begin{array}{l}7 / 4.7 \\
150 \\
(60-310)\end{array}$ & $\begin{array}{l}15 / 7.4 \\
203 \\
(114-335)\end{array}$ & $\begin{array}{l}7 / 6.1 \\
115 \\
(46-237)\end{array}$ \\
\hline Laboratories & $\begin{array}{l}\text { Obs/exp } \\
\text { SMR } \\
(95 \% \text { Cl) }\end{array}$ & $\begin{array}{l}8 / 11.6 \\
69 \\
(30-136)\end{array}$ & $\begin{array}{l}6 / 7.1 \\
84 \\
(31-184)\end{array}$ & $\begin{array}{l}0 / 0.8 \\
0 \\
(0-445)\end{array}$ & $\begin{array}{l}0 / 1.9 \\
0 \\
(0-200)\end{array}$ & $\begin{array}{l}14 / 4.3 \\
326 \\
(178-546)\end{array}$ & $\begin{array}{l}5 / 4.3 \\
117 \\
(38-274)\end{array}$ \\
\hline Other operations & $\begin{array}{l}\text { Obs/exp } \\
\text { SMR } \\
(95 \% \text { Cl) }\end{array}$ & $\begin{array}{l}20 / 24.3 \\
82 \\
(50-127)\end{array}$ & $\begin{array}{l}15 / 19.5 \\
77 \\
(43-127)\end{array}$ & $\begin{array}{l}2 / 1.4 \\
141 \\
(17-508)\end{array}$ & $\begin{array}{l}4 / 3.9 \\
102 \\
(28-261)\end{array}$ & $\begin{array}{l}6 / 8.7 \\
69 \\
(25-150)\end{array}$ & $\begin{array}{l}4 / 7.9 \\
51 \\
(14-131)\end{array}$ \\
\hline
\end{tabular}




\begin{tabular}{|c|c|c|c|c|c|c|c|}
\hline & & \multicolumn{3}{|c|}{ Lymphocytic leukaemia } & \multicolumn{3}{|c|}{ Myelogenous leukaemia } \\
\hline & & Total & Acute & Chronic & Total & Acute & Chronic \\
\hline Production & Obs/exp & $8 / 2.2$ & $0 / 0.4$ & $8 / 1.6$ & $4 / 3.4$ & $2 / 2.2$ & $2 / 1.0$ \\
\hline & $\begin{array}{l}\text { SMR } \\
(95 \% \mathrm{Cl})\end{array}$ & $\begin{array}{l}359 \\
(155-706)\end{array}$ & $\begin{array}{l}0 \\
(0-1030)\end{array}$ & $\begin{array}{l}497 \\
(215-980)\end{array}$ & $\begin{array}{l}118 \\
(32-302)\end{array}$ & $\begin{array}{l}93 \\
(11-335)\end{array}$ & $\begin{array}{l}202 \\
(24-728)\end{array}$ \\
\hline Production, coagulation & $\begin{array}{l}\text { Obs/exp } \\
\text { SMR } \\
(95 \% \mathrm{Cl})\end{array}$ & $\begin{array}{l}5 / 1.1 \\
452 \\
(147-1055)\end{array}$ & $\begin{array}{l}0 / 0.2 \\
0 \\
(0-2053)\end{array}$ & $\begin{array}{l}5 / 0.8 \\
607 \\
(197-1417)\end{array}$ & $\begin{array}{l}2 / 1.7 \\
116 \\
(14-420)\end{array}$ & $\begin{array}{l}1 / 1.1 \\
91 \\
(2-507)\end{array}$ & $\begin{array}{l}1 / 0.5 \\
188 \\
(5-1049)\end{array}$ \\
\hline Production, finishing & $\begin{array}{l}\text { Obs/exp } \\
\text { SMR } \\
(95 \% \mathrm{Cl})\end{array}$ & $\begin{array}{l}7 / 2.9 \\
238 \\
(96-491)\end{array}$ & $\begin{array}{l}0 / 0.6 \\
0 \\
(0-630)\end{array}$ & $\begin{array}{l}7 / 2.0 \\
344 \\
(138-709)\end{array}$ & $\begin{array}{l}7 / 4.9 \\
143 \\
(57-294)\end{array}$ & $\begin{array}{l}5 / 3.0 \\
165 \\
(54-386)\end{array}$ & $\begin{array}{l}2 / 1.6 \\
128 \\
(15-460)\end{array}$ \\
\hline Maintenance, shop & $\begin{array}{l}\text { Obs/exp } \\
\text { SMR } \\
(95 \% \mathrm{Cl})\end{array}$ & $\begin{array}{l}1 / 1.0 \\
98 \\
(2-545)\end{array}$ & $\begin{array}{l}0 / 0.2 \\
0 \\
(0-2256)\end{array}$ & $\begin{array}{l}0 / 0.7 \\
0 \\
(0-499)\end{array}$ & $\begin{array}{l}1 / 1.6 \\
63 \\
(2-350)\end{array}$ & $\begin{array}{l}0 / 1.0 \\
0 \\
(0-359)\end{array}$ & $\begin{array}{l}1 / 0.5 \\
216 \\
(5-1201)\end{array}$ \\
\hline Maintenance, field & $\begin{array}{l}\text { Obs/exp } \\
\text { SMR } \\
(95 \% \mathrm{Cl})\end{array}$ & $\begin{array}{l}2 / 3.0 \\
68 \\
(8-244)\end{array}$ & $\begin{array}{l}0 / 0.5 \\
0 \\
(0-815)\end{array}$ & $\begin{array}{l}2 / 2.1 \\
93 \\
(11-338)\end{array}$ & $\begin{array}{l}4 / 4.4 \\
92 \\
(25-234)\end{array}$ & $\begin{array}{l}2 / 2.8 \\
72 \\
(9-259)\end{array}$ & $\begin{array}{l}1 / 1.3 \\
79 \\
(2-440)\end{array}$ \\
\hline Labour, production & $\begin{array}{l}\text { Obs/exp } \\
\text { SMR } \\
(95 \% \text { Cl) }\end{array}$ & $\begin{array}{l}1 / 0.8 \\
132 \\
(3-736)\end{array}$ & $\begin{array}{l}0 / 0.1 \\
0 \\
(0-3032)\end{array}$ & $\begin{array}{l}1 / 0.5 \\
185 \\
(5-1031)\end{array}$ & $\begin{array}{l}2 / 1.2 \\
168 \\
(20-608)\end{array}$ & $\begin{array}{l}1 / 0.7 \\
143 \\
(4-795)\end{array}$ & $\begin{array}{l}1 / 0.4 \\
250 \\
(6-1390)\end{array}$ \\
\hline Labour, maintenance & $\begin{array}{l}\text { Obs/exp } \\
\text { SMR } \\
\text { (95\% CI) }\end{array}$ & $\begin{array}{l}4 / 1.8 \\
219 \\
(60-562)\end{array}$ & $\begin{array}{l}0 / 0.3 \\
0 \\
(0-1122)\end{array}$ & $\begin{array}{l}4 / 1.3 \\
309 \\
(84-792)\end{array}$ & $\begin{array}{l}6 / 2.9 \\
210 \\
(77-457)\end{array}$ & $\begin{array}{l}5 / 1.7 \\
295 \\
(96-688)\end{array}$ & $\begin{array}{l}1 / 1.0 \\
101 \\
(3-560)\end{array}$ \\
\hline Laboratories & $\begin{array}{l}\text { Obs/exp } \\
\text { SMR } \\
(95 \% \text { CI) }\end{array}$ & $\begin{array}{l}4 / 1.0 \\
390 \\
(106-999)\end{array}$ & $\begin{array}{l}0 / 0.2 \\
0 \\
(0-1796)\end{array}$ & $\begin{array}{l}4 / 0.7 \\
559 \\
(152-1431)\end{array}$ & $\begin{array}{l}6 / 1.8 \\
331 \\
(122-720)\end{array}$ & $\begin{array}{l}2 / 1.1 \\
177 \\
(22-642)\end{array}$ & $\begin{array}{l}3 / 0.6 \\
522 \\
(108-1526)\end{array}$ \\
\hline Other operations & $\begin{array}{l}\text { Obs/exp } \\
\text { SMR } \\
\text { (95\% CI) }\end{array}$ & $\begin{array}{l}1 / 2.2 \\
46 \\
(1-257)\end{array}$ & $\begin{array}{l}0 / 0.4 \\
0 \\
(0-1044)\end{array}$ & $\begin{array}{l}1 / 1.6 \\
64 \\
(2-354)\end{array}$ & $\begin{array}{l}3 / 3.3 \\
91 \\
(19-265)\end{array}$ & $\begin{array}{l}1 / 2.1 \\
48 \\
(1-269)\end{array}$ & $\begin{array}{l}2 / 1.0 \\
197 \\
(24-713)\end{array}$ \\
\hline
\end{tabular}

1992-98 (SMR $=98$, CI 89 to 107). Subjects had fewer than expected deaths from other major causes for the entire study period. Circulatory disease was the largest cause of death category, with 2932 observed and 3282 expected deaths $(\mathrm{SMR}=89$, CI 86 to 93$)$.

For the entire 1944-98 study period, there were fewer deaths than expected for each specific form of cancer, except for colorectal cancer $(S M R=109, C I 94$ to 125$)$, prostate cancer $(\mathrm{SMR}=104, \mathrm{CI} 88$ to 121$)$, Hodgkin's disease $(S M R=111, C I 58$ to 195$)$, and leukaemia $(S M R=116, C I$ 91 to 147). For NHL and multiple myeloma, respectively, SMRs were 100 (CI 75 to 130) and 95 (CI 62 to 140). Lung cancer (SMR $=91$, CI 84 to 99 ) accounted for $35 \%$ of all cancer deaths.

Table 3 presents further results for the 1944-98 follow up period for colorectal cancer, prostate cancer, Hodgkin's disease, multiple myeloma, leukaemia, and NHL. Table 4 presents results for 1968/1969-98 for specific forms of leukaemia.

Both ever hourly and never hourly subjects had slight increases in deaths from colorectal cancer and prostate cancer (table 3). Analyses by years since hire (a surrogate for induction time) and years worked (a surrogate for amount of exposure to chemicals in the industry) indicated that the SMR was increased for colorectal cancer in the subgroup of ever hourly men with 20-29 years since hire and with $10+$ years worked ( $S M R=147, C I 103$ to 205) and for prostate cancer in the subgroup with $30+$ years since hire and $<10$ years worked ( $S M R=155$, CI 113 to 206).

Never hourly subjects had an SMR above 100 and ever hourly subjects had an SMR below 100 for Hodgkin's disease (never hourly, SMR $=305$, CI 99 to 711; ever hourly, $\mathrm{SMR}=77, \mathrm{CI} 31$ to 158 ) and multiple myeloma (never hourly, SMR = 146, CI 54 to 317; ever hourly, SMR $=86$, CI 53 to 133). The SMRs did not display any marked patterns by years since hire and years worked for either of these two cancers.
Ever hourly subjects had more than expected leukaemia deaths (63/51, SMR $=123$, CI 94 to 157) and NHL deaths (49/ $44, \mathrm{SMR}=111, \mathrm{CI} 82$ to 147 ), whereas never hourly subjects had fewer than expected deaths from both diseases (leukaemia: 8/9.8, SMR = 82; NHL: 4/9.1, SMR =44). The leukaemia excess was highest in the subgroup of ever hourly men with 20-29 years since hire and 10+ years worked ( SMR $=258$, CI 156 to 403). The SMR for NHL was highest, but was not statistically significant, in the same subgroup (SMR $=170, \mathrm{CI}$ 85 to 305 ).

Analyses of specific forms of leukaemia, with the restricted time period of follow up, included 65 (92\%) of the 71 leukaemia deaths and $49(92 \%)$ of the 53 NHL deaths that occurred during the total follow up period of 1944-98. For all subjects included in the time restricted analyses, the SMR was 126 (CI 97 to 161) for all forms of leukaemia combined, 128 (CI 77 to 200) for lymphocytic leukaemia, 127 (CI 84 to 183) for myelogenous leukaemia, and 123 (CI 73 to 194) for other and unspecified forms of leukaemia (table 4). Of the 19 lymphocytic leukaemias, 16 were chronic ( SMR $=151$, CI 87 to 247), one was acute ( $\mathrm{SMR}=42$, CI 1 to 234), and two were not specified as acute or chronic ( $\mathrm{SMR}=107, \mathrm{CI} 13$ to 387 ). Of the 28 myelogenous leukaemias, 14 were acute $(\mathrm{SMR}=102, \mathrm{CI} 56$ to 171$), 11$ were chronic $(\mathrm{SMR}=167, \mathrm{CI}$ 83 to 299), and three were not specified as acute or chronic $(\mathrm{SMR}=173, \mathrm{CI}=36-505)$. Other and unspecified leukaemias included one monocytic leukaemia, 11 acute leukaemias of unspecified cell type, and six leukaemias that were not specified by cell type or as acute or chronic.

Hourly workers had an overall leukaemia SMR of 135 (CI 103 to 175) for the 1968-98 time period (data not displayed in a table) and had an increased SMR for each specific form of leukemia analysed, except for acute lymphocytic and acute myelogenous leukaemias (table 4). Analyses of specific forms of leukaemia by years since hire and years worked among hourly subjects indicated that in the group with 20-29 years since hire and $10+$ years worked, showed that the overall 
excess of leukaemia in 1968/1969-98 in this group $(\mathrm{SMR}=284$, CI 168 to 449$)$ was not limited to a specific type. There was a non-statistically significant excess of lymphocytic leukaemia (4/1.7, SMR $=233$, CI 65 to 597 and a statistically significant excess of myelogenous leukaemia $(9 / 2.8$, SMR $=320$, CI 146 to 607$)$, and results were strongest for chronic myelogenous leukaemia $(6 / 0.9, \mathrm{SMR}=655, \mathrm{CI}$ 240 to 1426). The SMR for chronic lymphocytic leukaemia was increased in the groups with 20-29 years since hire and with $30+$ years since hire, but these results were based on small numbers.

Analyses by work area found small increases in colorectal cancer among some subgroups of production and maintenance workers, whereas workers in laboratories and other operations had deficits of deaths from this form of cancer (table 5). Workers in maintenance shop had the highest SMR for colorectal cancer ( SMR $=142$, CI 81 to 231). Maintenance labourers had the highest SMR for prostate cancer (31 observed/24 expected, SMR $=128$, CI 87 to 181). Observed numbers of prostate cancer deaths were either close to or below the expected numbers of deaths in the other work areas. Mortality from multiple myeloma or Hodgkin's disease was not strikingly increased in any specific work area.

Leukaemia mortality was associated with work in polymerisation $(S M R=204$, CI 121 to 322$)$, coagulation $(S M R=231$, CI 111 to 425), maintenance labour $(S M R=203$, CI 114 to 335), and laboratories (SMR $=326$, CI 178 to 546) (table 5). NHL deaths were increased in most of the work areas, but none of the increases was striking. All but two of the 10 subjects who had leukaemia and who worked in coagulation had also worked in polymerisation, whereas 10 of the 18 leukaemia decedents in polymerisation had never worked in coagulation. The highest NHL SMRs were in finishing $(\mathrm{SMR}=143, \mathrm{CI} 82$ to 233$)$ and production labour (SMR $=157$, CI 43 to 403$)$.

Analyses of specific forms of leukaemia by work area found that the SMRs for all lymphocytic leukaemia and for chronic lymphocytic leukaemia were increased more than twofold in polymerisation, coagulation, finishing, maintenance labour, and laboratories (table 6). There was considerable overlap between subjects in polymerisation and finishing, as well as between polymerisation and coagulation, as already mentioned. Analyses of mutually exclusive work area groups were imprecise. Subjects employed in polymerisation but never in finishing had more than expected deaths from chronic lymphocytic leukaemia (2/0.7). Subjects employed in finishing but never in polymerisation had equal observed and expected numbers of chronic lymphocytic leukaemia deaths (1/1.1).

Work area specific analyses were largely uninformative for myelogenous leukaemia because of small numbers. The only strong associations were for laboratory work and chronic myelogenous leukaemia (3/0.6, SMR $=522$, CI 108 to 1526) and for maintenance labour and acute myelogenous leukaemia $(5 / 1.7, \operatorname{SMR}=295$, CI 96 to 688$)$.

Period of hire analyses indicated that the excess of leukaemia in the overall study group was concentrated among men hired in the 1950s (31/21, SMR $=150$, CI 101 to 211) (data not presented in a table). Men hired in the 1940s had 28/30 leukaemia deaths ( $S M R=93$, CI 62 to 134), and men hired in or after 1960 had 12/10 leukaemia deaths $(S M R=120, C I 62$ to 210$)$. Although men hired in the 1940s had nearly equal observed and expected leukaemia deaths, the subgroup with 20-29 years since hire and 10+ years worked had a twofold increase $(7 / 3.5, \mathrm{SMR}=202$, CI 81 to 416). The small increase among men hired in or after 1960 was concentrated in the subgroup with 20-29 years since hire and $<10$ years worked (5/1.6, SMR $=313$, CI 101 to 729$)$.

\section{DISCUSSION}

The update added to our previous study of synthetic rubber industry workers 83401 (18\% increase) person-years of follow up, $1578(34 \%)$ total deaths, 492 (44\%) total cancer deaths, 20 (39\%) leukaemia deaths, 20 (61\%) NHL deaths, and six $(30 \%)$ multiple myeloma deaths. The subjects had an overall mortality rate $14 \%$ lower than expected on the basis of the state or provincial general population rates, and their mortality rate from all cancers combined was $8 \%$ lower than expected. Subjects' rates of circulatory, non-malignant respiratory, digestive, and genitourinary disease deaths and of deaths from external causes also were lower than expected. These deficits tended to diminish as time since hire increased, suggesting the gradual disappearance of the healthy worker effect. Synthetic rubber industry workers, overall or certain subgroups, had more than expected deaths from several forms of cancer, including leukaemia, NHL, multiple myeloma, and cancers of the colorectum and prostate.

\section{Leukaemia}

The total group of 17924 subjects had 16\% more than expected leukaemia deaths. This increase was small and was not limited to a single form of leukaemia. It was concentrated in long term hourly workers, and particularly in men who had worked in SBR related polymerisation, coagulation, maintenance labour, and laboratory jobs. In each of these work groups, potential for exposure to agents of interest was relatively high. It is reasonable to assume that ever hourly workers, because of their more direct contact with plant operations, had higher potential for exposure to chemicals in the workplace than did never hourly workers. Polymerisation and some laboratory jobs entailed regular exposure to butadiene and styrene monomers and some exposure to DMDTC. Coagulation workers could have been exposed to all three agents, particularly when monomer stripping and recovery operations were inefficient. Maintenance labourers also could have had high exposure during reactor vessel and other cleaning operations. In contrast, hourly workers in finishing, skilled maintenance, and other plant areas such as warehouses, utilities, and offices had less potential for regular exposure to butadiene, although some field maintenance operations may have entailed high exposure to monomers.

Data on specific forms of leukaemia were difficult to interpret because of imprecision due to small numbers. Chronic lymphocytic leukaemia was increased in all work areas having potentially high exposure to butadiene, styrene, and DMDTC (polymerisation, coagulation, maintenance labour, and laboratories). Chronic myelogenous leukaemia mortality was substantially increased only for laboratory workers, whereas results for other work areas were based on very small numbers. Analyses of acute myelogenous leukaemia mortality by work area were imprecise because of small numbers and found a substantially increased SMR only among maintenance labourers.

Assuming that exposures in the synthetic rubber industry cause leukaemia, it is of interest that patterns of leukaemia excesses by years since hire and years worked varied to some extent according to the specific form of leukaemia. Chronic myelogenous leukaemia and chronic lymphocytic leukaemia accounted largely for the overall leukaemia excess in the subgroup with 20-29 years since hire and 10+ years worked. Acute myelogenous leukaemia and other leukaemia also were increased in this category, but the latter results were based on small numbers. Chronic lymphocytic leukaemia, but not chronic myelogenous leukaemia, was increased both in the subgroup with 20-29 years since hire and 10+ years worked and in subgroups with $30+$ years since hire, a pattern that 
may reflect the fact that survival is longer for chronic lymphocytic leukaemia than for acute or chronic myelogenous leukaemias. If exposures in the synthetic rubber industry cause chronic lymphocytic leukaemia and chronic myelogenous leukaemia, the induction period appears to be 20 or more years. An excess of acute myelogenous leukaemia occurred in relatively short term employees with $<20$ years since hire. This excess could be due to chance or confounding. It is also possible that the acute myelogenous leukaemia has a shorter induction time than chronic lymphocytic leukaemia or chronic myelogenous leukaemia.

The results of the present study for leukaemia are consistent with those reported earlier by Santos-Burgoa, ${ }^{7}$ by Matanoski et al, ${ }^{89}$ and by us. ${ }^{124}$ The increases in leukaemia that we observed in the overall subject group (16\%) and in hourly workers $(23 \%)$ were similar to the leukaemia SMR of 129 found in the recent study of butadiene monomer production workers. ${ }^{10}$ The latter investigation reported patterns of leukaemia mortality by duration of employment and work category that suggested that the small increase was not due to agents in the workplace. However, direct estimates of butadiene exposure were not available for analysis. The Divine and Hartman ${ }^{10}$ and other studies of butadiene exposed workers have not investigated specific forms of leukaemia. The mechanism by which butadiene could cause leukaemia in humans is not understood, and research on the relation between exposure to butadiene and biomarkers of genotoxicity in humans has not yielded consistently positive results. ${ }^{12}$

Studies of reinforced plastics workers who were exposed to styrene concentrations much higher than levels typically found in the synthetic rubber industry and who were not exposed to butadiene have not reported any consistent relation with leukaemia. ${ }^{13-20}$ Matanoski et al did not find that styrene was associated with leukaemia in their analyses. ${ }^{9}$

No other epidemiological study has investigated the relation between DMDTC and leukaemia or other LHC. However, because DMDTC is an immune system depressant, it is plausible that it could increase the risk of NHL and of some leukaemias. ${ }^{21}$

Uncertainty remains about the specific agent(s) and about the role of unidentified confounding factors that might be responsible for the association found between employment in the synthetic rubber industry and leukaemia in this study. The carcinogenic mechanisms through which butadiene, styrene, or DMDTC could cause leukaemia in humans have not been established, and external epidemiological support for a leukaemogenic role is limited for these agents. ${ }^{22-25}$

\section{NHL, multiple myeloma, and Hodgkin's disease}

There was no excess of NHL deaths among the overall study group. Small increases in NHL deaths occurred among subgroups (polymerisation, maintenance labour, and laboratories) having potentially high exposure to butadiene, styrene, and DMDTC, but none of these increases was large or statistically significant. Workers in coagulation, another area with potential high exposure to the monomers, had no excess of this form of cancer. NHL SMRs did not display any marked patterns by years since hire or years worked.

Matanoski et al, ${ }^{9}$ in an investigation that included many of the same subjects in our study, reported a positive association between styrene and NHL. However, their findings and that of the present study are inconsistent with other research. Notably, studies of occupational groups exposed to levels of styrene higher than those found in the synthetic rubber industry have not reported any consistent increase in NHL deaths or cases. ${ }^{13-20} 24$

Matanoski et al reported that multiple myeloma was associated with butadiene in a study that included many of the subjects in our study. ${ }^{9}$ Our analyses by work area/job group provided little support for a causal relation between synthetic rubber industry employment and multiple myeloma. The highest work area/job group specific SMR for multiple myeloma was for production labourers. Maintenance labourers had an SMR of 150, but this result was based on only seven observed and 4.7 expected deaths. Data on polymerisation, coagulation, and laboratory workers were sparse but did not suggest any positive associations. Divine and Hartman ${ }^{10}$ found slightly more than expected deaths from multiple myeloma among butadiene production workers, but like that study's results for leukaemia, the data on multiple myeloma were too sparse and too internally inconsistent to provide any substantial support for a causal interpretation.

Hodgkin's disease was not strikingly increased in any specific work area. Our study included only 12 decedents with Hodgkin's disease. This number was too small for detailed analyses.

\section{Colorectal and prostate cancer}

Some subgroups of synthetic rubber industry workers had more than expected deaths from these cancers. However, in our companion study, we did not find any relation between cumulative exposure estimates to butadiene, styrene, or DMDTC and these forms of cancer. Chance or uncontrolled confounding by non-occupational risk factors for these two cancers could be responsible for the patterns that we observed.

\section{Strengths and limitations}

The subjects included in our study constituted a large group with many years of follow up and reasonably complete vital status ascertainment. The update added considerably to the amount of information available for assessing mortality patterns and used expanded and refined exposure estimates. Limitations included the lack of information on potential non-occupational confounders, such as diet or physical activity. Another limitation was the lack of information on the specific form of leukaemia for 18 of 65 (28\%) of the leukaemia decedents. Another paper will report the results of a review of the medical records of the LHC decedents. We did not use the medical record information in the present study in order to maintain comparability with the general population, for which death rates are based on underlying cause of death as recorded on death certificates, without medical record review.

Work history information received from the study plants could not have depended on vital status or cause of death, as the information predated the occurrence of death. We developed work history classifications independent of knowledge of subjects' cause of death. Comparisons between the study group and the general population were based on the underlying cause of death data from death certificates. It is reasonable to assume that errors in diagnosing and recording leukaemia as a cause of death were similar in synthetic rubber workers and the general population at large, as knowledge on the potential association between workers in the industry and lymphohaematopoietic cancers was limited. Study methods should have minimised the potential for nonrandom misclassification of subjects by exposure and disease. However, such misclassification still could have occurred, particularly in subgroups that entailed small numbers of subjects and decedents. The potential of losses to follow up to produce erroneous results was small. Assuming that all subjects lost to follow up actually survived through the end of 1998, their lost person-years accounted for only 3\% of the total person-years in the study. Analytic procedures adjusted for known determinants for leukaemia rates-that is, age, 
calendar year, and race. Thus, confounding by these factors is unlikely to account for the positive association. However, we cannot rule out the possibility that the association we observed is due to unidentified confounding factors.

\section{CONCLUSIONS}

The study found that some subgroups of synthetic rubber industry workers had an excess of mortality from leukaemia that may have been due to workplace exposure. Uncertainty remains about the specific agent(s) that might be responsible for the observed excesses and about the role of unidentified confounding factors. Some subgroups of synthetic rubber industry workers had more than expected deaths from colorectal cancer and prostate cancer. These increases did not appear to be related to occupational exposure in the industry.

\section{Authors' affiliations}

N Sathiakumar, M Macaluso, R Matthews, E Delzell, Department of Epidemiology, Ryals School of Public Health, University of Alabama at Birmingham, Birmingham, AL, USA

J Graff, Wayne State University School of Medicine, Detroit, MI, USA G Maldonado, University of Minnesota, School of Public Health, Division of Environmental Health Sciences, Minneapolis, MN, USA, Sponsor: Health Effects Institute

\section{REFERENCES}

1 Delzell E, Sathiakumar N, Hovinga M, et al. A follow-up study of synthetic rubber workers. Toxicology 1996;113:182-9.

2 Macaluso M, Larson R, Delzell $E$, et al. Leukemia and cumulative exposure to butadiene, styrene and benzene among workers in the synthetic rubber industry. Toxicology 1996;113:190-202.

3 Sathiakumar N, Delzell E, Hovinga M, et al. Mortality from cancer and other causes of death among synthetic rubber workers. Occup Environ Med 1998;55:230-5

4 Delzell E, Macaluso M, Sathiakumar N, et al. Leukemia and exposure to 1,3-butadiene, styrene and dimethyldithiocarbamate among workers in the synthetic rubber industry. Chemico-Biological Interactions 2001;135136:515-34

5 Marsh GM, Youk AO, Stone RA, et al. OCMAP-Plus: a program for the comprehensive analysis of occupational cohort data. J Occup Env Med 1998:40:351-62.

6 Harris NL, Jaffe ES, Diebold J, et al. World Health Organization classification of neoplastic diseases of the hematopoietic and lymphoid tissues: report of the Clinical Advisory Committee Meeting - Airlie House, Virginia, November 1997. J Clin Oncol 1999;17:3835-49.
7 Santos-Burgoa C, Matanoski GM, Zeger S, et al. Lymphohematopoietic cancer in styrene-butadiene polymerization workers. Am J Epidemiol 1992;136:843-54.

8 Matanoski GM, Elliot E, Tao X, et al. Lymphohematopoietic cancers in butadiene and styrene exposure in synthetic rubber manufacture. Am NY Acad Sci 1997;837:157-69.

9 Matanoski GM, Schwartz L. Mortality of workers in styrene-butadiene polymer production. J Occup Med 1987;29:675-80.

10 Divine BJ, Hartman CM. A cohort mortality study among workers at a 1,3butadiene facility. Chemico-Biological Interactions 2001;135-136:535-53.

11 Divine BJ, Hartman CM. Mortality update of butadiene production workers. Toxicology 1996;1 13:169-81.

12 Albertini RJ, Sram RJ, Vacek PM, et al. Biomarkers for assessing occupational exposures to 1,3-butadiene. Chemico-Biological Interactions 2001;135136:429-53.

13 Nicholson WJ, Selikoff IJ, Seidman H. Mortality experience of styrenepolystyrene polymerization workers. Initial findings. Scand J Work Environ Health 1978;4:247-52.

14 Hodgson JT, Jones RD. Mortality of styrene production, polymerization and processing workers at a site in northwest England. Scand J Work Environ Health 1985; 11:347-52

15 Bond GG, Bodner KM, Olsen GW, et al. Mortality among workers engaged in the development or manufacture of styrene-based products - an update. Scand J Work Environ Health 1992;18:145-54.

16 Kogevinas M, Ferro G, Saracci R, et al. Cancer mortality in an international cohort of workers exposed to styrene. In: Sorsa M, Peltonen K, Vainio H, Hemminki K, eds. Butadiene and styrene: assessment of health hazards. IARC Scientific Publications No 127. Lyon, France: WHO, IARC, 1993:289-300.

17 Coggon D. Epidemiological studies of styrene-exposed populations. Crit Rev Toxicol 1994;24:S107-S115.

18 Kogevinas M, Ferro G, Andersen A, et al. Cancer mortality in a historical cohort study of workers exposed to styrene. Scand J Work Environ Health 1994;20:251-61.

19 Kolstad HA, Lynge E, Olsen J, et al. Incidence of lymphohematopoietic malignancies among styrene-exposed workers of the reinforced plastics industry. Scand J Work Environ Health 1994;20:272-8.

20 Kolstad HA, Juel K, Olsen J, et al. Exposure to styrene and chronic health effects. Occup Environ Med 1995;52:320-7.

21 Melbye M, Trichopoulos D. Non-Hodkgin's lymphoma. In: Adami H-O Hunter D, Trichopoulos D, eds. Textbook of cancer epidemiology. New York: Oxford University Press, 2002:546.

22 International Agency for Research on Cancer. 13-Butadiene. In IARC Monographs on the Evaluation of Carcinogenic Risks to Humans. Vol 71. Reevaluation of some organic chemicals, hydrazine and hydrogen peroxide. Lyons, France: WHO, IARC, 1999:109-225.

23 Cohen JT, Carlson G, Charnley G, et al. (Panel Report). A comprehensive evaluation of the potential health risks associated with occupational and environmental exposure to styrene. J Toxicol Envr Health B Crit Rev 2002;5:1-265.

24 International Agency for Research on Cancer Styrene. IARC Monographs on the Evaluation of Carcinogenic Risks to Humans. Vol 82. Some traditional herbal medicines, some mycotoxins, naphthalene and styrene. Lyons, France: WHO, IARC, 2002:437-550.

25 Irons RD, Stillman WS, Pyatt DW, et al. Comparative toxicity of dithiocarbamates and butadiene metabolites in human lymphoid and bone marrow cells. Chem Biol Interact 2001;135-136:615-25. 